\title{
Recycling of Waste Rubber by Thermo-Mechanical Treatment in a Twin-Screw Extruder ${ }^{+}$
}

\author{
Łukasz Zedler, Paulina Kosmela, Adam Olszewski, Paulina Burger, Krzyszfot Formela and \\ Aleksander Hejna * \\ Department of Polymer Technology, Gdańsk University of Technology, Narutowicza 11/12, 80-233 Gdańsk, \\ Poland; lukasz.zedler@pg.edu.pl (Ł.Z.); paulina.kosmela@pg.edu.pl (P.K.); \\ adam.olszewski@student.pg.edu.pl (A.O.); paulina_anna_burger@interia.pl (P.B.); krzform1@pg.edu.pl (K.F.) \\ * Correspondence: aleksander.hejna@pg.gda.pl; Tel.: +48-58-347-2234 \\ + Presented at the First International Conference on “Green" Polymer Materials 2020, 5-25 November 2020; \\ Available online: https://cgpm2020.sciforum.net/.
}

Published: 4 November 2020

\begin{abstract}
Recycling of waste tires is a significant issue considering both environmental and economic aspects. One of the leading recycling routes is the shredding of tires resulting in the generation of ground tire rubber. This material can be easily introduced into various polymer matrices as a filler, reducing the use of conventionally applied petroleum-based materials. In such cases, it is essential to ensure sufficient interfacial compatibility, which could be achieved by the proper modification of the rubber surface. Different methods of treatment of ground tire rubber aim to activate its surface and introduce functional groups, which could provide the possibility for interfacial interactions and incorporation of the significant amounts of recycled material. Therefore, in the presented paper, we examined the impact of thermo-mechanical treatment in twin-screw extruder on the appearance and chemical structure of ground tire rubber. Moreover, for each set of process parameters, the specific mechanical energy required for the processing was calculated, which could provide essential insights for the potential industrial application of the analyzed process. The energy demand should be considered as a very important issue during development of "greener" processes and materials.
\end{abstract}

Keywords: ground tire rubber; rubber modification; surface activation; specific mechanical energy; chemical structure

\section{Introduction}

The utilization of post-consumer car tires is a critical environmental issue. Among the recycling methods, there are two, which are definitely the most popular: energy recovery and material recycling. In general, the European Union's primary method is material recycling (stands for $\sim 40 \%$ of car tires recycling). However, in some countries, e.g., Poland, this process still gives way to incineration. Nevertheless, it only allows for recovery of up to $40 \%$ of the energy used to produce a new tire [1]. Energy recovery can be considered a relatively safe process, but it must be performed under very controlled conditions and using special equipment. Therefore, soon, especially considering current pro-environmental trends and law regulations, incineration should be rather replaced by material recycling [2].

Material recycling converts post-consumer tires into different products, which could be further applied in different industrial processes. The most popular is the shredding of tires, resulting in ground tire rubber (GTR). Its properties could be regulated by the proper selection of the shredding method and adjustment of its parameters. Nevertheless, it allows for obtaining secondary materials with high efficiency and good quality products. 
Due to car tires' excellent mechanical properties, ground tire rubber can be considered auspicious material, which can be applied as a filler for polymer composites [3]. Its application in polymer technology was already reviewed [4]. It perfectly fits into current trends associated with the circular economy approach to polymer technology and looking for the possibilities to introduce various by-products and waste materials into polymer matrices. Incorporation of GTR into polymer matrices could significantly reduce the use of the virgin polymers reducing the use of petroleum and simultaneously decrease the environmental footprints of final products [5]. From the economic point of view, most polymers are obtained from raw materials of petrochemical origin, so the plastic market is strongly dependent on oil prices, which is a raw material with decreasing availability and unstable price. Therefore, the search for alternative sources of raw materials that can be used to produce plastic products is fully justified. Application of ground tire rubber, low-cost by-product could noticeably reduce the cost of the final polymer products. Nevertheless, to fully exploit ground tire rubber's mechanical properties, it is often essential to modify it. Such an approach is the answer for one of the most significant issues related to the polymer/ground tire rubber composites-insufficient interfacial compatibility, which does not allow to use of GTR as real reinforcement for polymer matrices [6]. Numerous methods of ground tire rubber modification have been developed and described in the literature [7-9]. They included mechanical, thermomechanical, and mechanical-chemical treatments and other methods using microwave radiation, ultrasounds, microorganisms, or $\mathrm{CO}_{2}$ in the supercritical state [10]. Unfortunately, most modification methods described in the literature are based on periodical processes, limiting their industrial applications. On the other hand, continuous processes ensure greater efficiency, reduce the amount of waste generated (both auxiliary materials, such as solvents, and waste generated during starting and stopping the process). The economic aspect of the process is also positively influenced. Moreover, in terms of filler treatment, it is necessary to adjust its properties, hence the process's parameters, to the final use of filler because different polymer matrices require different modifications. Therefore, the effective process applied for the modification of fillers should be characterized by the continuous character and easiness in adjusting its parameters. A potentially very effective method is the modification of fillers using reactive extrusion. This process is fast, relatively cheap, flexible, and considered a pro-ecological alternative to traditional, periodical modification [11]. The reactive extrusion enables the precise regulation of its parameters such as temperature in individual zones of the cylinder, screw speed, capacity, material residence time in the extruder, and finally, the size of shear forces acting on the material regulated by changing the screw configuration. As a result, adjustment of the whole process for the desired product is relatively easy and fast.

Having in mind the advantages of the reactive extrusion, in the presented research work, we aimed to investigate its application in recycling ground tire rubber aimed at the manufacturing of potential filler for foamed polyurethane matrix. Thermo-mechanical treatment of GTR was applied, assisted by adding two types of rapeseed oil, fresh one, and post-consumer waste from the restaurant. The process's energetic efficiency was evaluated, and the changes in the chemical structure of modified GTR.

\section{Experimental}

\subsection{Materials}

Ground tire rubber obtained by ambient grinding of used tires (a combination of passenger car and truck tires in 50:50 mass ratio), whose average particle size is approximately $0.8 \mathrm{~mm}$ was produced and provided by Recykl S.A. (Śrem, Poland).

Two types of rapeseed oil were applied as modifiers for ground tire rubber. Fresh rapeseed oil was acquired from Lidl (Poland), while waste oil was obtained from a local restaurant (Gdańsk, Poland).

During the evaluation of the chemical structure of modified GTR following chemicals were applied: acetone, dibutylamine, chlorobenzene, hydrochloric acid, toluene diisocyanate (TDI), and 
$3^{\prime}, 3^{\prime \prime}, 5^{\prime}, 5^{\prime \prime}$-tetrabromophenolsulfonphthalein. All chemicals were acquired from Sigma Aldrich (Poland) and were used as received.

\subsection{Thermo-Mechanical Treatment of Ground Tire Rubber}

Thermo-mechanical treatment of GTR was performed with EHP 2x20 Sline co-rotating twinscrew extruder from Zamak Mercator (Poland). The extruder has nine heating/cooling zones with a screw diameter of $20 \mathrm{~mm}$ and an L/d ratio of 40 . Ground tire rubber was premixed with $20 \mathrm{or} 40 \mathrm{phr}$ (parts per hundred of rubber) of oil before treatment and then was dosed into the extruder by a volumetric feeder with a constant throughput of $2 \mathrm{~kg} / \mathrm{h}$. The screw speed was set at 50, 150, or 350 $\mathrm{rpm}$. The value of $50 \mathrm{rpm}$ was selected as a minimum screw speed, which could be applied to process the ground tire rubber effectively. Nevertheless, for samples containing $40 \mathrm{phr}$ of oils, minimum screw speed had to be increased to $150 \mathrm{rpm}$ to enable the extrusion without clogging in the dosing section. Barrel temperature in all zones was set at $200{ }^{\circ} \mathrm{C}$. For each set of parameters, extrusion was carried out for at least $5 \mathrm{~min}$ after stabilizing the extruder's motor load, indicating the stabilization process.

\subsection{Measurements}

To evaluate the effect of barrel temperature on the progress of ground tire rubber reclamation, the specific mechanical energy $(\mathrm{SME}$, in $\mathrm{kWh} / \mathrm{kg}$ ) was determined. SME was calculated using Equation (1):

$$
\mathrm{SME}=\mathrm{N} / \mathrm{Q}
$$

where: $\mathrm{N}$ - the consumption of drive motor power $(\mathrm{kW}), \mathrm{Q}$ - throughput $(\mathrm{kg} / \mathrm{h})$.

For calculations of the specific mechanical energy, the average motor load from at least $20 \mathrm{~min}$ of extrusion was used. Moreover, the total energy consumption (TEC) of the extruder was monitored.

Changes in the chemical structure of ground tire rubber were evaluated using a modified method for the determination of free isocyanate groups content by titration with dibutylamine, according to ASTM D-2572. The $0.5 \mathrm{~g}$ samples of GTR were put in a glass flask with $0.5 \mathrm{~g}$ of toluene diisocyanate and $20 \mathrm{~cm}^{3}$ of acetone. Mixtures were thoroughly mixed, sealed, and stored at room temperature for $24 \mathrm{~h}$. Then, proper amounts of dibutylamine solution in chlorobenzene and $3^{\prime}, 3^{\prime \prime}, 5^{\prime}, 5^{\prime \prime}$-tetrabromophenolsulfonphthalein were added. Then, mixtures were titrated with $0.1 \mathrm{M}$ hydrochloric acid until the color change to yellow. Obtained results were compared with the free isocyanate content of neat toluene diisocyanate to determine the number of functional groups at the rubber surface able to react with isocyanates. Such evaluation is essential for the potential application of modified GTR as a filler for polyurethane materials.

The free isocyanate content of the GTR/TDI mixture was calculated according to the following Equation (2):

$$
\%_{\mathrm{NCO}}=\left(4.202 \cdot\left(\mathrm{V}_{\mathrm{B}}-\mathrm{Vs}\right) \cdot \mathrm{NHCl}\right) / \mathrm{mTDI}
$$

where: $\mathrm{V}_{\mathrm{B}}$ - the volume of $\mathrm{HCl}$ required for titration of the blank sample, $\mathrm{ml}$; $\mathrm{V}_{\mathrm{s}}$ - the volume of $\mathrm{HCl}$ required for titration of analyzed sample, $\mathrm{ml} ; \mathrm{NHCl}-$ molarity of $\mathrm{HCl}, \mathrm{M} ; \mathrm{mTDI}$ - the mass of TDI placed in the flask, g.

Based on these values, the assumed hydroxyl numbers (LоH) of ground tire rubber were calculated. During calculations, it was assumed that all of the consumed isocyanate groups reacted with the GTR particles. Another assumption was that all of the functional groups present on the surface of GTR were hydroxyls. Considering these assumptions, the number of hydroxyl groups, which took part in reactions was calculated following the Equation (3):

$$
\mathrm{X}_{\mathrm{OH}}=\mathrm{X}_{\mathrm{NCO}}=\left((\% \mathrm{NCO}-\mathrm{TDI}-\% \mathrm{NCO}) \cdot \mathrm{mTDI}_{\mathrm{T}} \cdot 2\right) /\left(\mathrm{MTDI}_{\mathrm{TD}} \cdot 100\right)
$$

where: \%NCO-TDI - free isocyanate content in TDI, equal to $42.7 \%$; MTDI - the molar mass of TDI, equal to $174.2 \mathrm{~g} / \mathrm{mol}$.

Then, the hydroxyl number of ground tire rubber was calculated from the Formula (4): 
where: mGTR - the mass of GTR placed in the flask, g.

\section{Results}

In Table 1, there are presented results obtained during the modification of ground tire rubber in a twin-screw extruder. From the industrial point of view, an essential aspect of all processes included in the production cycle is energy consumption, which directly impacts the cost-efficiency of production. Therefore, it is essential to determine the amount of energy required by different processes. It can be seen that the increase of the screw speed resulted in the noticeable reduction of the motor load and reduced the specific mechanical energy required for the processing of a given mass of material. It was related to the shortened residence time of material in the extruder barrel, as well as the smaller level of filling of the working volume by the material [12]. Nevertheless, a higher amount of energy needed by the motor to turn the screws caused a slight $6 \%$ increase in total energy consumption.

The motor load, hence the specific mechanical energy, was noticeably decreased when oils were introduced. Such an effect was associated with the lubricating properties of oils, which reduced the friction between ground tire rubber particles and between rubber particles and the extruder barrel. Moreover, oils could swell the rubber particles, reducing their stiffness, resulting in lower friction inside the extruder barrel. Total energy consumption was also slightly reduced from 0.160 to 0.157 $\mathrm{kWh} / \mathrm{kg}$ of processed GTR. The increase in oil content caused a slight rise in total energy consumption. However, specific mechanical energy was maintained at the same level of 0.006 $\mathrm{kWh} / \mathrm{kg}$ of material for the screw speed of $350 \mathrm{rpm}$.

Considering the type of applied oil, it can be seen that the introduction of waste oil reduced the specific mechanical energy comparing to the neat rapeseed oil. Such an effect could be associated with the partial decomposition of oil during cooking. It probably resulted in the generation of lowermolecular weight products, which acted as plasticizers during the process and reduced the friction inside the extruder barrel. Moreover, they could be more effective in swelling rubber particles, especially for the longer residence time. The most significant difference between oils was noted for samples extruded with a screw speed of $50 \mathrm{rpm}$, so when residence time was noticeably the highest.

Table 1. Results obtained during thermo-mechanical treatment of ground tire rubber.

\begin{tabular}{|c|c|c|c|c|c|c|c|c|}
\hline Sample & Oil Content, phr & Screw Speed, rpm & Motor Load, \% & SME, kWh/kg & $\mathrm{TEC}, \mathrm{kWh} / \mathrm{kg}$ & $\%$ NCO, $\%$ & $\Delta_{\mathrm{NCO}}, \%$ & $\begin{array}{l}\text { Lон } \mathrm{mg} \\
\mathrm{KOH} / \mathrm{g}\end{array}$ \\
\hline Neat GTR & - & - & - & - & - & 33.3 & 9.4 & 61.7 \\
\hline \multirow{2}{*}{ GTR } & \multirow[b]{2}{*}{-} & 50 & 33.0 & 0.053 & 0.160 & 36.3 & 6.4 & 41.1 \\
\hline & & 350 & 2.2 & 0.006 & 0.170 & 32.1 & 10.6 & 67.8 \\
\hline \multirow{4}{*}{ Rapeseed oil } & \multirow{2}{*}{20} & 50 & 21.3 & 0.033 & 0.157 & 37.9 & 4.8 & 30.8 \\
\hline & & 350 & 3.5 & 0.006 & 0.173 & 33.8 & 8.9 & 56.9 \\
\hline & \multirow{2}{*}{40} & 150 & 4.6 & 0.008 & 0.165 & 36.7 & 6.0 & 38.3 \\
\hline & & 350 & 3.6 & 0.006 & 0.175 & 34.5 & 8.2 & 53.1 \\
\hline \multirow{4}{*}{ Waste oil } & \multirow{2}{*}{20} & 50 & 16.4 & 0.026 & 0.157 & 32.4 & 10.3 & 66.6 \\
\hline & & 350 & 3.6 & 0.006 & 0.173 & 30.9 & 11.8 & 75.2 \\
\hline & \multirow{2}{*}{40} & 150 & 4.7 & 0.008 & 0.168 & 29.9 & 12.8 & 82.3 \\
\hline & & 350 & 3.6 & 0.006 & 0.178 & 30.9 & 11.8 & 75.9 \\
\hline
\end{tabular}

As mentioned in the introduction, the presented work aimed to manufacture the modified ground tire rubber, which could be further applied as a filler for the foamed polyurethane matrix. One of the main components used during polyurethane synthesis is isocyanates. Due to the presence of free isocyanate groups, they are very reactive with compounds containing various functional groups, including amines, alcohols, or carboxylic acids. Some of these groups, especially hydroxyl and carboxyl, may be present on the surface of ground tire rubber after thermo-mechanical treatment. As a result, GTR can interact with isocyanates during the manufacturing of polyurethane foam and affect the polymerization reactions [13]. Therefore, it is essential to adjust the foams' formulations when ground tire rubber is applied as a filler [14]. Nevertheless, for proper adjustment, it is crucial 
to determine the portion of the functional groups present on the surface of GTR particles and able to interact with isocyanate groups. To do so, we modified the method for the determination of free isocyanate content according to the ASTM D-2572 standard.

In Table 1, there are presented the free isocyanate contents (\%NCO) of GTR/TDI mixtures and the decrease of their value comparing to neat TDI $\left(\Delta_{\mathrm{NCO}}\right)$. It can be seen that neat ground tire rubber, before the extrusion, already contains functional groups on the surface, which can react with isocyanate. The content of free isocyanate groups was $33.3 \%$, comparing to $42.7 \%$ for neat TDI. These functional groups are generated during shredding of tires, conducted under air atmosphere, enabling partial oxidation of rubber particles. The extrusion treatment of GTR caused changes in the chemical structure of the material. When a lower screw speed of $50 \mathrm{rpm}$ was applied, the calculated hydroxyl value of rubber was noticeably decreased, which indicated the lowered amount of hydroxyl groups at the surface of particles. The adverse effect was noted for the screw speed of $350 \mathrm{rpm}$ when the LOH increase was noted. It can be associated with the shorter residence time of material inside the extruder barrel and the lower extent of its decomposition, as well as lower shear forces acting on the material (smaller filling of the working volume).

The introduction of oils caused noticeable differences in the chemical structure of GTR. When fresh rapeseed oil was added, the hydroxyl values were reduced. It can be associated with the hydrophobic character of oil, hence its low hydroxyl value. Hartman et al. [15] reported the hydroxyl value of rapeseed oil of $16.4 \mathrm{mg} \mathrm{KOH} / \mathrm{g}$. However, it may be even lower, depending on the oil quality. Similar to the extrusion treatment of neat GTR without oil, higher screw speeds resulted in higher hydroxyl values. It is also related to the shorter residence time and lower extent of rubber swelling with oil. Such an effect was not noted for the $40 \mathrm{phr}$ content of waste oil.

On the other hand, the application of waste oil increased hydroxyl values. It was associated probably with the partial decomposition of oil during its use in the restaurant. Its stability was lower, which resulted in burning during processing. According to the literature data, the rapeseed oil smoke point may vary in the range of $180-220^{\circ} \mathrm{C}$ [16]. Therefore, oil with previous thermal history could be easily oxidized and degraded during processing, which resulted in the generation of lower-molecular weight compounds with functional groups during processing.

\section{Conclusions}

Obtained results related to ground tire rubber's applied treatment indicate that the extrusion should be considered an up-and-coming method for modifying this by-product. It enables the accessible tailoring of the final product properties by adjusting process parameters or introducing additional modifiers. It is also exciting considering the potential applications of modified GTR as a filler for foamed polyurethane matrices. Thermo-mechanical treatment may be applied to activate the surface of rubber particles and increase the number of functional groups, which is crucial for developing polyurethane-based materials. Furthermore, this study shows that it is possible to process GTR continuously, obtaining a reclaimed product which is also in a powdery form. This allows us to conclude that it is possible to obtain products based on reclaimed GTR, using multi-component injection molding. This method, according to the authors, is the future of the processing of waste rubber products, and this study opens new possibilities in this area.

The proposed method for determining the hydroxyl value of modified rubber particles could also be considered very interesting from the polyurethane point of view. Proper design of polyurethane formulations should include the presence of all functional groups, especially hydroxyls, able to react with isocyanates. Such an approach enables the effective development of polyurethane structures and strong interfacial interactions with applied filler, which is crucial for the satisfactory mechanical performance of composite material.

Future works related to the extrusion treatment of ground tire rubber, considering its potential applications in polyurethane materials, should include:

- Examining the broader range of the extrusion parameters could potentially result in higher hydroxyl values of modified ground tire rubber and enhance the interfacial interactions with the 
polyurethane matrix. Moreover, modified GTR could be applied as a cross-linking agent for rigid polyurethane materials when sufficiently high values would be achieved.

- Incorporation of the additional modifiers, which could enhance the interfacial adhesion with the polyurethane matrix.

- Investigation of the volatile organic compounds emissions during modification, especially when additional modifiers are applied, which is essential for developing the truly environmentallyfriendly processes.

- Reduction of the environmental impacts of the process, e.g., by lowering the process temperature or other adjustments of process parameters aimed at reducing the energy demand.

Author Contributions: Ł.Z., K.F. and A.H. conceived and designed the experiments; Ł.Z., P.K., A.O. and P.B. performed the experiments; Ł.Z., P.K. and A.H. analyzed the data; Ł.Z. and K.F. contributed reagents/materials/analysis tools; A.H. wrote the paper. All authors have read and agreed to the published version of the manuscript.

Acknowledgments: This work was supported by The National Centre for Research and Development (NCBR, Poland) in the frame of LIDER/3/0013/L-10/18/NCBR/2019 project-Development of technology for the manufacturing of foamed polyurethane-rubber composites for the use as damping materials.

Conflicts of Interest: The authors declare no conflict of interest.

\section{References}

1. Laboy-Nieves, E.N. Energy Recovery from Scrap Tires: A Sustainable Option for Small Islands like Puerto Rico. Sustainability 2014, 6, 3105-3121, doi:10.3390/su6053105.

2. Godlewska, J. Recovery and Recycling of Waste Tires in Poland. Procedia Eng. 2017, 182, 229-234, doi:10.1016/j.proeng.2017.03.173.

3. Zedler, Ł.; Przybysz-Romatowska, M.; Haponiuk, J.; Wang, S.; Formela, K. Modification of Ground Tire Rubber-Promising Approach for Development of Green Composites. J. Compos. Sci. 2020, 4, 2, doi:10.3390/jcs4010002.

4. Ramarad, S.; Khalid, M.; Ratnam, C.T.; Luqman Chuah, A.; Rashmi, W. Waste tire rubber in polymer blends: A review on the evolution, properties and future. Prog. Mater. Sci. 2015, 72, 100-140, doi:10.1016/j.pmatsci.2015.02.004.

5. Korol, J.; Hejna, A.; Burchart-Korol, D.; Wachowicz, J. Comparative Analysis of Carbon, Ecological, and Water Footprints of Polypropylene-Based Composites Filled with Cotton, Jute and Kenaf Fibers. Materials 2020, 13, 3541, doi:10.3390/ma13163541.

6. Hernández, E.H.; Gámez, J.F.H.; Cepeda, L.F.; Muñoz, E.J.C.; Corral, F.S.; Rosales, S.G.S.; Velázquez, G.N.; Morones, P.G.; Martínez, D.I.S. Sulfuric acid treatment of ground tire rubber and its effect on the mechanical and thermal properties of polypropylene composites. J. Appl. Polym. Sci. 2017, 134, 44864, doi:10.1002/app.44864.

7. Li, Y.; Shen, A.; Lyu, Z.; Wang, S.; Formela, K.; Zhang, G. Ground tire rubber thermo-mechanically devulcanized in the presence of waste engine oil as asphalt modifier. Constr. Build. Mater. 2019, 222, 588600, doi:10.1016/j.conbuildmat.2019.06.162.

8. Aoudia, K.; Azem, S.; Ait Hocine, N.; Gratton, M.; Pettarin, V.; Seghar, S. Recycling of waste tire rubber: Microwave devulcanization and incorporation in a thermoset resin. Waste Manage. 2017, 60, 471-481, doi:10.1016/j.wasman.2016.10.051.

9. Cheng, X.; Long, D.; Huang, S.; Li, Z.; Guo, X. Time effectiveness of the low-temperature plasma surface modification of ground tire rubber powder. J. Adhes. Sci. Technol. 2015, 29, 1330-1340, doi:10.1080/01694243.2015.1026958.

10. Colom, X.; Faliq, A.; Formela, K.; Canavate, J. FTIR spectroscopic and thermogravimetric characterization of ground tyre rubber devulcanized by microwave treatment. Polym. Test. 2016, 52, 200-208, doi:10.1016/j.polymertesting.2016.04.020.

11. Formela, K.; Klein, M.; Colom, X.; Saeb, M.R. Investigating the combined impact of plasticizer and shear force on the efficiency of low temperature reclaiming of ground tire rubber (GTR). Polym. Degrad. Stabil. 2016, 125, 1-11, doi:10.1016/j.polymdegradstab.2015.12.022. 
12. Kelly, A.L.; Brown, E.C.; Coates, P.D. The effect of screw geometry on melt temperature profile in single screw extrusion. Polym. Eng. Sci. 2006, 46, 1706-1714, doi:10.1002/pen.20657.

13. Piszczyk, Ł.; Hejna, A.; Danowska, M.; Strankowski, M.; Formela, K. Polyurethane/ground tire rubber composite foams based on polyglycerol: Processing, mechanical and thermal properties. J. Reinf. Plast. Compos. 2015, 34, 708-717, doi:10.1177\%2F0731684415579089.

14. Formela, K.; Hejna, A.; Zedler, Ł.; Przybysz, M.; Ryl, J.; Saeb, M.R.; Piszczyk, Ł. Structural, thermal and physico-mechanical properties of polyurethane/brewers' spent grain composite foams modified with ground tire rubber. Ind. Crop. Prod. 2017, 108, 844-852, doi:10.1016/j.indcrop.2017.07.047.

15. Hartman, L.; Lago, R.C.A.; Azeredo, L.C.; Azeredo, M.A.A. Determination of hydroxyl value in fats and oils using an acid catalyst. The Analyst 1987, 112, 145, doi:10.1039/an9871200145.

16. Skrökki, A. Quality of Frying Oils in Grill Restaurants and Catering Establishments. Fat Sci. Technol. 1995, 97, 299-301, doi:10.1002/lipi.19950970716.

Publisher's Note: MDPI stays neutral with regard to jurisdictional claims in published maps and institutional affiliations.

(C) 2020 by the authors. Submitted for possible open access publication under the terms and conditions of the Creative Commons Attribution (CC BY) license (http://creativecommons.org/licenses/by/4.0/). 